\title{
Extension to de Broglie formula of Quantum Mechanics
}

\section{Zhong-Yue Wang}

Engineering Department, SBSC, No.580 Songhuang Road ,Shanghai 201703, China

E-mail address: zhongyuewang@ymail.com

Keywords: de Broglie relation; momentum operator; vacuum Cherenkov effect; Casimir effect;

\section{ABSTRACT:}

Planck-Einstein's $E=\hbar \omega$ and de Broglie's $\mathbf{p}=\hbar \mathbf{k}$ are two fundamental equations of quantum physics. But vacuum Cherenkov radiation (VCR) is convincing evidence that the momentum of a single photon should be $\mathbf{p}=\hbar \boldsymbol{\beta}$ where the phase constant $\boldsymbol{\beta}$ takes the place of the wave vector $\mathbf{k}$. Apart from the momentum, the operator $-i \hbar \nabla$ is associated to $m_{0} c$. The acousto-optic effect and anomalous VCR can be applied to test this conjecture.

PACS: $\quad$ 03.65.Ta; 42.50.Pq; 41.60.Bq; 84.40.Fe; 14.80.Bn

\section{INTRODUCTION}

The key of quantum mechanics is the Hamiltonian in terms of the momentum operator $\hat{p} \rightarrow-i \hbar \nabla$ which associated with de Broglie formula $p=\hbar k$. There are lots of experimental verifications for $p=\hbar k$ [1]

\section{Traveling waves}

Compton effect, 1923

Davisson and Germer 1927, Thomson 1928

Estermann and Stern, 1930

\section{Stationary waves}

Density of states

Bohr-Sommerfeld quantization

In de Broglie's theory, the phase velocity is $V_{p}=\frac{\omega}{k}$ and group velocity is $V_{g}=\frac{d \omega}{d k}$. Nevertheless, they are $V_{p}=\frac{\omega}{\beta}$ and $V_{g}=\frac{d \omega}{d \beta}$ in microwave electronics where the phase constant $\beta$ is a component of $k$ and the phase is changed merely in this direction. The wave with a phase velocity $\frac{\omega}{\beta}<c$ is called slow wave [2][3] and $\frac{\omega}{\beta}>c$

Dispersion relation

[4][5] is fast wave. Concretely,

Phase velocity

Free space $\omega=k c$

$$
\frac{\omega}{k}=c
$$

Slow wave $\omega=k c=\sqrt{\beta^{2} c^{2}-\tau^{2} c^{2}}<\beta c \quad(\tau$-transverse eigenvalue $)[2][3] \quad \frac{\omega}{\beta}<c$

Fast wave $\quad \omega=k c=\sqrt{\beta^{2} c^{2}+\omega_{c}^{2}}>\beta c \quad\left(\omega_{c}\right.$-cutoff frequency $) \quad$ [4][5] $\frac{\omega}{\beta}>c$

Then whether the momentum should be $p=\hbar \beta$ under the circumstances? 


\section{CASIMIR EFFECT}

The ratio of the Poynting vector(energy flux density) $\mathbf{S}=\mathbf{E} \times \mathbf{H}$ to momentum density $\mathbf{g}=\varepsilon_{0} \mathbf{E} \times \mathbf{B}$ of an electromagnetic field in vacuum is a constant [6][7]

$$
\frac{\mathbf{S}}{\mathbf{g}}=\frac{\mathbf{E} \times \mathbf{H}}{\varepsilon_{0} \mathbf{E} \times \mathbf{B}}=\frac{1}{\varepsilon_{0} \mu_{0}}=c^{2}
$$

An EM field consists of photons so the relation between the energy and momentum of a single photon should be

$$
\frac{\frac{\bar{w}}{\bar{g}}}{\frac{\bar{g}}{N}}=\frac{\bar{w}}{\bar{g}}=\frac{\overline{\bar{g}}}{\frac{\bar{S}}{\bar{w}}}=\frac{c^{2}}{\overline{\bar{S}}}=\frac{c^{2}}{V_{e}}
$$

where $w$ is the energy density, $N$ is the number density of photons and $V_{e}=\frac{\bar{S}}{\bar{w}}$ is the energy velocity of this field. In a hollow waveguide, the energy velocity is $c \sqrt{1-\frac{\omega_{c}^{2}}{\omega^{2}}}<c$ [8] and the ratio of energy to momentum equals

$$
\frac{c^{2}}{V_{e}}=\frac{c^{2}}{c \sqrt{1-\frac{\omega_{c}^{2}}{\omega^{2}}}}=\frac{c}{\sqrt{1-\frac{\omega_{c}^{2}}{\omega^{2}}}}=V_{p}=\frac{\omega}{\beta}=\frac{\hbar \omega}{\hbar \beta}
$$

In the Casimir effect [9] [12], two metal plates can be regarded as a one-dimensional rectangular waveguide and experiments demonstrate the Planck-Einstein relation $E=\hbar \omega$ in this structure. Consequently, the momentum of a photon in the waveguide should be $\hbar \beta$ rather than $\hbar k$.

\section{NORMAL VCR}

Cherenkov radiation is generally emitted by charged particles pass through the dielectric medium at a speed $V$ faster than the phase velocity $\frac{1}{\sqrt{\varepsilon \mu}}<c$. Owing to wave-particle duality, this effect can also be deduced from quantum mechanics [13]. Moreover, it occurs in vacuum $\left(\frac{\omega}{k}=\frac{1}{\sqrt{\varepsilon_{0} \mu_{0}}}\right)$ provided that the phase velocity is decreased by slow wave systems [14] and we use the quantum theory to study too. Consider charged particles moving near the surface of a periodic structure [2][3] (Fig.1) 


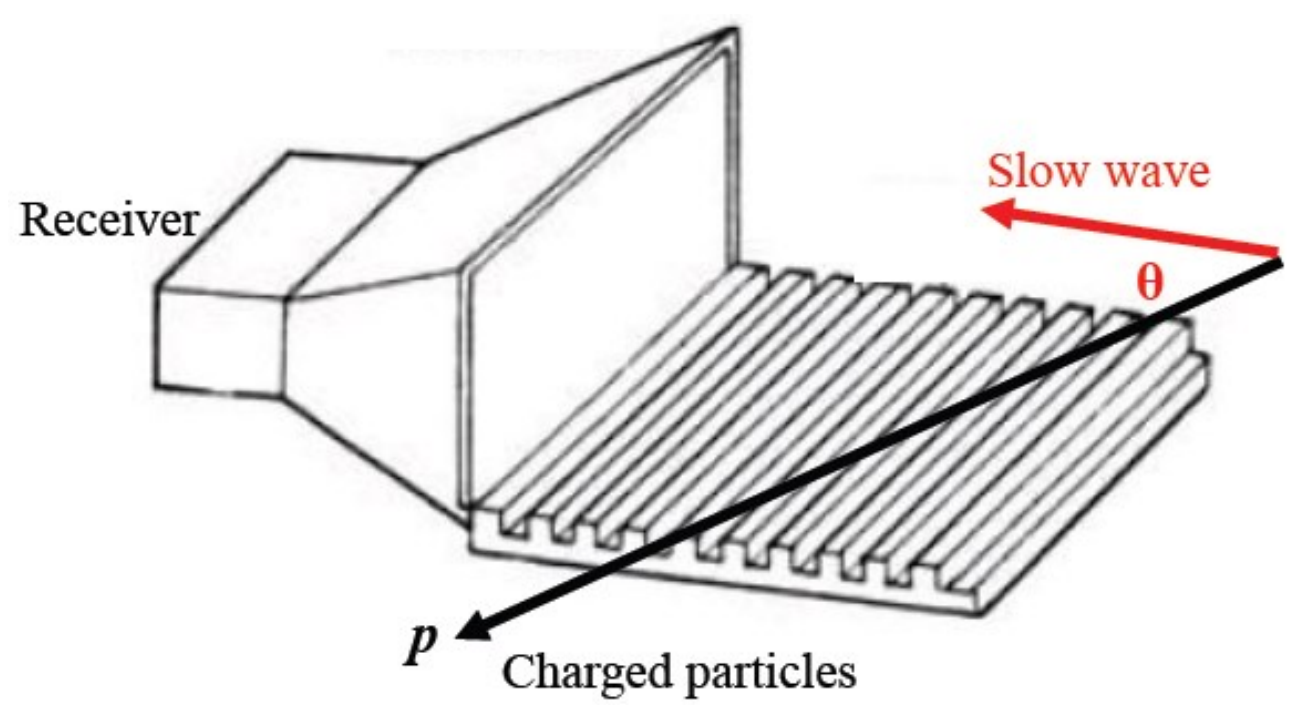

Figure 1. Corrugated planar conductor

Assume the momentum of a photon is $\hbar \mathbf{k}$ (Fig.2),

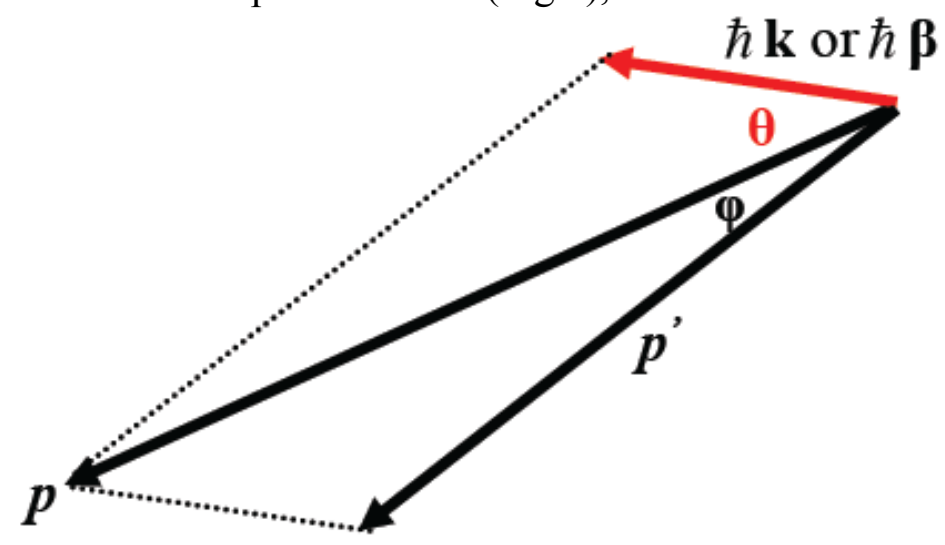

Figure 2. Parallelogram law

Momentum conservation (Fig.2):

$$
\begin{gathered}
\mathbf{p}-\hbar \mathbf{k}=\mathbf{p}^{\prime} \\
p^{2}-2 p \hbar k \cos \theta+\hbar^{2} k^{2}=p^{\prime 2} \\
\cos \theta=\frac{p^{2}+\hbar^{2} k^{2}-p^{\prime 2}}{2 p \hbar k}
\end{gathered}
$$

Energy conservation:

The energy of a charged particle is $\sqrt{p^{2} c^{2}+m_{0}^{2} c^{4}}$,

$$
\begin{gathered}
\sqrt{p^{2} c^{2}+m_{0}^{2} c^{4}}-\hbar \omega=\sqrt{p^{\prime 2} c^{2}+m_{0}^{2} c^{4}} \\
p^{2} c^{2}+m_{0}^{2} c^{4}-2 \hbar \omega E+\hbar^{2} \omega^{2}=p^{\prime 2} c^{2}+m_{0}^{2} c^{4} \\
p^{2}-\frac{2 \hbar \omega E}{c^{2}}+\frac{\hbar^{2} \omega^{2}}{c^{2}}=p^{\prime 2}
\end{gathered}
$$

Substituting Eq.(10) into Eq.(6),

$$
\cos \theta=\frac{\frac{2 \hbar \omega E}{c^{2}}-\frac{\hbar^{2} \omega^{2}}{c^{2}}+\hbar^{2} k^{2}}{2 p \hbar k}=\frac{\frac{2 \hbar \omega E}{c^{2}}}{2 p \hbar k}=\frac{\frac{\omega}{k}}{\frac{p c^{2}}{E}}
$$


Since $E=\sqrt{p^{2} c^{2}+m_{0}^{2} c^{4}}=\frac{m_{0} c^{2}}{\sqrt{1-\frac{V^{2}}{c^{2}}}}$ and $p=\frac{m_{0} V}{\sqrt{1-\frac{V^{2}}{c^{2}}}}$ of the charged particle,

$$
\begin{gathered}
\frac{p c^{2}}{E}=V \\
\cos \theta=\frac{\frac{\omega}{k}}{V}=\frac{c}{V}>1
\end{gathered}
$$

VCR is forbidden because of $\frac{\omega}{k} \equiv c$ in free space, fast wave structures [4][5] and slow wave systems [2][3]. However, people utilize slow wave devices in vacuum to generate VCR [14]. To explain this phenomenon, the momentum of a photon must be $\hbar \boldsymbol{\beta}$. Hence

$$
\begin{aligned}
& \mathbf{p}-\hbar \boldsymbol{\beta}=\mathbf{p}^{\prime} \\
& \cos \theta=\frac{p^{2}+\hbar^{2} \beta^{2}-p^{\prime 2}}{2 p \hbar \beta} \\
& \sqrt{p^{2} c^{2}+m_{0}^{2} c^{4}}-\hbar \omega=\sqrt{p^{\prime 2} c^{2}+m_{0}^{2} c^{4}} \\
& \cos \theta=\frac{\frac{2 \hbar \omega E}{c^{2}}-\frac{\hbar^{2} \omega^{2}}{c^{2}}+\hbar^{2} \beta^{2}}{2 p \hbar \beta}=\frac{\frac{\omega}{\beta}}{\frac{p c^{2}}{E}}+\frac{\hbar^{2}\left(\beta^{2}-\frac{\omega^{2}}{c^{2}}\right)}{2 p \hbar \beta}
\end{aligned}
$$

The rightmost term including a reduced Planck constant $\hbar$ has no counter part in classical physics. In the main, it has negligible effect on the trajectory because $\hbar \beta$ of a photon is much less than the momentum $p$ of the charged particle, i.e.

$$
\begin{gathered}
\cos \theta \approx \frac{\frac{\omega}{\beta}}{\frac{p c^{2}}{E}}=\frac{\frac{\omega}{\beta}}{V} \\
\cos \varphi=\frac{p^{2}+p^{\prime 2}-\hbar^{2} \beta^{2}}{2 p p^{\prime}} \approx \frac{p^{2}+p^{\prime 2}}{2 p p^{\prime}} \approx 1, \quad \varphi \approx 0
\end{gathered}
$$

Now we interpret why VCR merely exists in a slow wave device under conditions of $\frac{\omega}{\beta}<V<c$ and cannot be found in free space or fast-wave systems $\left(\frac{\omega}{\beta}>c\right)$. Besides mentioned periodic structures, VCR can be excited by a smooth surface such as the dielectric waveguide [15] and Sommerfeld wire [16] [18]. They're useful to obtain THz waves.

\section{ANOMALOUS VCR}

In addition to normal VCR observed long ago[14], there should be anomalous VCR when the quantum correction plays a major role. For instance, the phase constant of a corrugated plane surface is [2][3]

$$
\beta=\frac{\omega}{c} \sqrt{1+\tan ^{2} \frac{\omega}{c} d}=\frac{\omega}{c \cos \frac{\omega}{c} d}>\frac{\omega}{c} \quad(d \text { corrugation depth })
$$

In event of $\frac{\omega}{c} d \rightarrow \frac{\pi}{2}, \hbar \beta>>\frac{\hbar \omega}{c}$ is of the same order of magnitude of $p$ and Eq.(16) is now 


$$
\cos \theta \approx \frac{\frac{\omega}{\beta}}{V}+\frac{\hbar \beta}{2 p}
$$

Meanwhile, the phase velocity $\frac{\omega}{\beta}$ is very low and $\frac{\frac{\omega}{\beta}}{V} \approx 0$. Thus,

$$
\cos \theta \approx \frac{\hbar \beta}{2 p}
$$

Namely radiation whose angular frequency is about $\frac{\pi c}{2 d}$ has an enormous phase constant $\beta=\frac{2 p \cos \theta}{\hbar} \rightarrow \infty$ and

$$
\cos \varphi=\frac{p^{2}+p^{\prime 2}-\hbar^{2} \beta^{2}}{2 p p^{\prime}} \approx \frac{p^{2}+p^{\prime 2}-(2 p \cos \theta)^{2}}{2 p p^{\prime}}
$$

On the other hand, it is $p^{\prime} \approx p$ due to Eq.(8) and $\hbar \omega \approx \hbar \frac{\pi c}{2 d}<<\sqrt{p^{2} c^{2}+m_{0}^{2} c^{4}}$. Accordingly,

$$
\begin{aligned}
\cos \varphi \approx \frac{2 p^{2}-(2 p \cos \theta)^{2}}{2 p^{2}} & =1-2 \cos ^{2} \theta=-\cos 2 \theta \\
\text { i.e. } \varphi & =\pi-2 \theta
\end{aligned}
$$

That is to say, a small portion of charged particles are deflected through a large angle $\varphi=\pi-2 \theta($ Fig. 3$)$

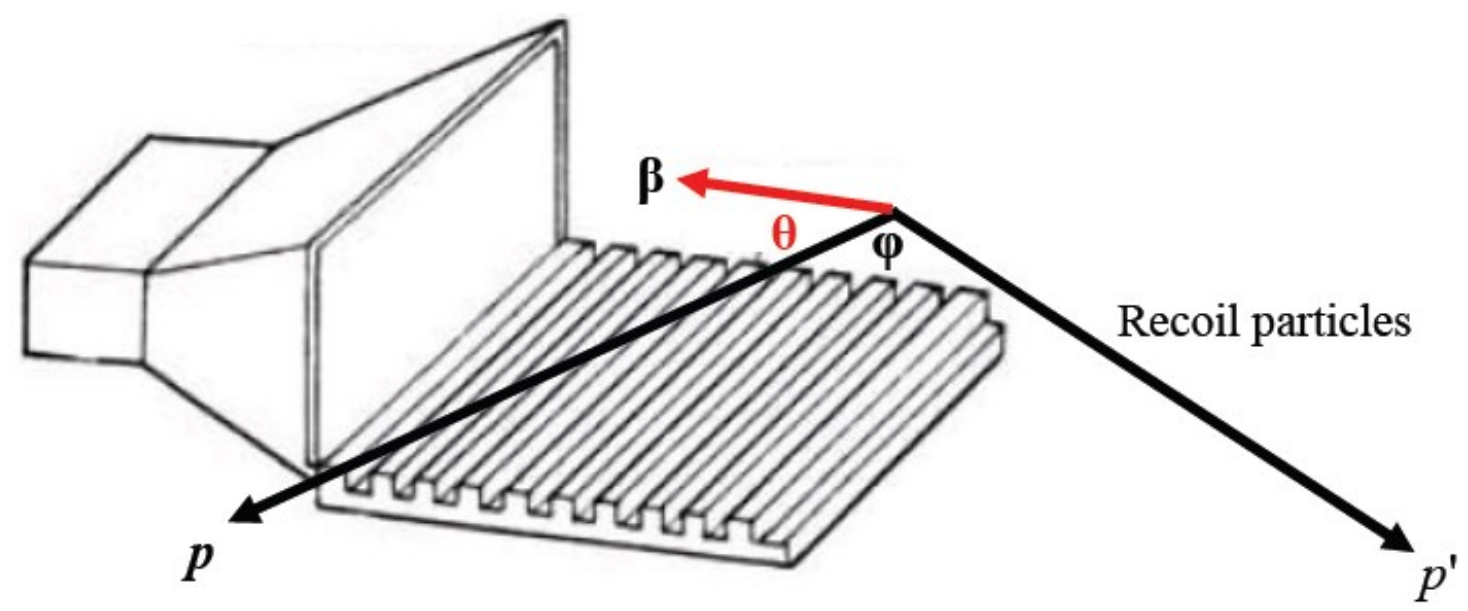

Figure 3. Anomalous VCR

In particular, the particle will be defected backwards $(\varphi \approx \pi)$ if $\theta \approx 0$. Anomalous radiation is a purely quantum effect and cannot be predicted by classical electrodynamics. 


\section{ACOUSTO-OPTIC INTERACTION}

The light can be diffracted by sound waves through acousto-optic devices [19] (Fig.4).

\section{Sound waves}

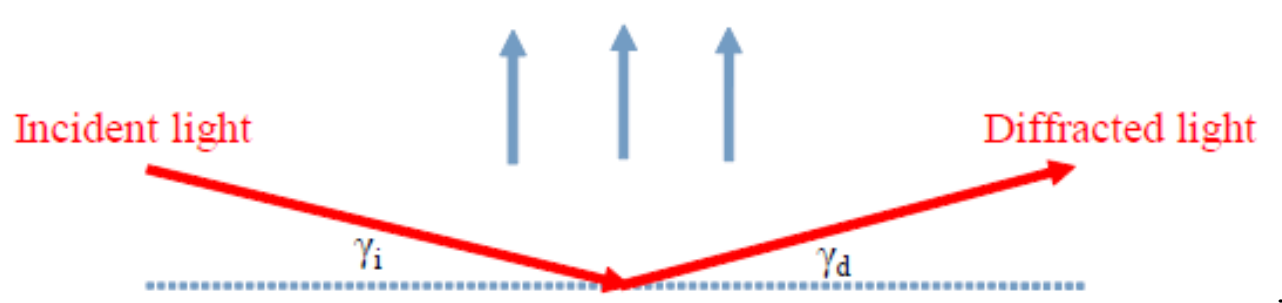

Figure 4. Bragg Diffraction

The momentum $P_{s}$ of a phonon is much less that of the incident photon $p_{i}$ and diffracted photon $p_{d}$ (Fig.5).

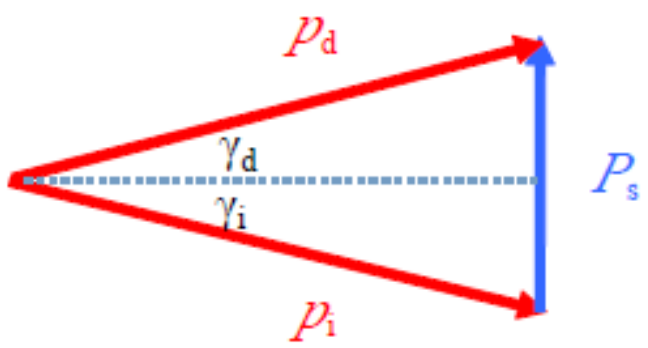

Figure 5. Momentum conservation

$$
\begin{gathered}
P_{s}<<p_{i} \\
p_{i} \approx p_{d} \\
\text { Bragg angle } \sin \gamma_{i}=\frac{P_{s}}{2 p_{i}}
\end{gathered}
$$

In unbonuded acousto-optic materials,

$$
\sin \gamma_{i}=\frac{P_{s}}{2 p_{i}}=\frac{\hbar K_{s}}{2 \hbar k_{i}}=\frac{K_{s}}{2 k_{i}}
$$

As bounded materials in different structures, there may be $P_{s}=\hbar \beta_{s} \neq \hbar K_{s}$ and $p_{i}=\hbar \beta_{i} \neq \hbar k_{i}$ for sound waves and EM waves.

\section{PHYSICAL MEANING}

The rectangular waveguide [4][5] can be regarded as an infinite square well [20] of photons and the wave function is

$$
\Psi_{(x, y, z)}=0 \quad\left(|x| \geq \frac{a}{2},|y| \geq \frac{b}{2}\right)
$$

Stationary wave equation:

$$
\nabla^{2} \Psi+k^{2} \Psi=0
$$

Waves propagate along the $z$-axis,

Separation of variables

$$
\Psi_{(x, y, z)}=\psi_{(x, y)} e^{i \beta z} \quad\left(i=\sqrt{-1}, \beta=k_{z}\right)
$$

$$
\begin{aligned}
& \frac{d^{2} X}{d x^{2}}+k_{x}^{2} X=0 \\
& \frac{d^{2}}{d y^{2}} Y+k_{y}^{2} Y=0
\end{aligned}
$$


On account of the boundary condition (29),

$$
\begin{gathered}
k_{x}=l_{x} \frac{\pi}{a} \quad\left(l_{x}=0,1,2,3 \ldots . . .\right) \\
k_{y}=l_{y} \frac{\pi}{b} \quad\left(l_{y}=0,1,2,3 \ldots \ldots\right) \\
k_{x}^{2}+k_{y}^{2}=\pi^{2}\left(\frac{l_{x}^{2}}{a^{2}}+\frac{l_{y}^{2}}{b^{2}}\right)=\frac{\omega_{c}^{2}}{c^{2}} \quad[4][5]
\end{gathered}
$$

Transverse components of the momentum of a single photon are not

$$
\begin{gathered}
p_{x}=\hbar k_{x} \\
p_{y}=\hbar k_{y} \\
\sqrt{p_{x}^{2}+p_{y}^{2}}=\sqrt{\hbar^{2}\left(k_{x}^{2}+k_{y}^{2}\right)}=\frac{\hbar \omega_{c}}{c}
\end{gathered}
$$

otherwise the total momentum of this photon should be

$$
\sqrt{p_{z}^{2}+p_{x}^{2}+p_{y}^{2}}=\sqrt{\hbar^{2} \beta^{2}+\hbar^{2}\left(k_{x}^{2}+k_{y}^{2}\right)}=\hbar \sqrt{\beta^{2}+\frac{\omega_{c}^{2}}{c^{2}}}=\hbar k
$$

Ratio of energy to momentum

$$
\frac{\hbar \omega}{\hbar k}=\frac{\omega}{k}=c
$$

Eqs.(37) (39) are inconsistent with the result of classical electrodynamics that transverse components of the Poynting vector and momentum density are zero [21].To resolve this question, $p_{x}$ and $p_{y}$ have to be zero although $k_{x}$ and $k_{y}$ could be non-zero. Therefore,

$$
\begin{aligned}
& \text { Wave number } \quad k=\sqrt{\beta^{2}+k_{x}^{2}+k_{y}^{2}}=\sqrt{\beta^{2}+\frac{\omega_{c}^{2}}{c^{2}}}=\frac{\omega}{c}>\beta \\
& \text { Momentum } \quad p=\sqrt{p_{x}^{2}+p_{y}^{2}+p_{z}^{2}}=\sqrt{\hbar^{2} \beta^{2}+0+0}=\hbar \beta<\hbar k
\end{aligned}
$$

Ratio of energy to momentum

$$
\frac{\hbar \omega}{\hbar \beta}=\frac{\omega}{\beta}=V_{p}>c
$$

Operators $-i \hbar \frac{\partial}{\partial x}$ and $-i \hbar \frac{\partial}{\partial y}$ result in rest mass multiplied by $c$.

$$
\begin{gathered}
\hbar k_{x}=\hbar l_{x} \frac{\pi}{a}=m_{0 x} c \\
\hbar k_{y}=\hbar l_{y} \frac{\pi}{b}=m_{0 y} c \\
l_{x} \neq 0 \text { and } l_{y} \neq 0 \quad-T M \text { modes } \\
l_{x}=0, l_{y} \neq 0 \text { or } l_{x} \neq 0, l_{y}=0-T E \text { modes } \\
\omega_{c}=\sqrt{k_{x}^{2}+k_{y}^{2}} c=\pi c \sqrt{\frac{l_{x}^{2}}{a^{2}}+\frac{l_{y}^{2}}{b^{2}}}=\frac{m_{0} c^{2}}{\hbar}
\end{gathered}
$$

A non-zero rest mass of $T E$ and $T M$ wave is not surprising, if we think of longitudinal oscillations $\mathbf{B}_{z} \neq 0$ and $\mathbf{E}_{z} \neq 0$ in the direction of wave propagation. The quantity $m_{0}=\frac{\hbar \omega_{c}}{c^{2}}$ derives from massless Maxwell's equations and the value is zero in an infinitely large waveguide $(a \rightarrow \infty, b \rightarrow \infty)$ equivalent to free space. This has nothing to do with massive Proca's equations [24] whose rest mass of a photon is still nonzero even in free space and does not violate any fundamental physical law such as gauge invariance and Coulomb's inverse square law [25]. 
In sum, the dispersion relation of electromagnetic waves in a hollow waveguide

$$
\omega^{2}=\underbrace{\beta^{2} c^{2}+\omega_{c}^{2}}_{k^{2} c^{2}}
$$

corresponds to the relativistic mechanical equation

$$
E^{2}=p^{2} c^{2}+m_{0}^{2} c^{4}
$$

Total energy

$$
E=\hbar \omega
$$

Momentum

$$
p=\hbar \beta
$$

Rest mass

Mechanical velocity

$$
m_{0}=\frac{\hbar \omega_{c}}{c^{2}}
$$

Phase velocity

$$
\begin{gathered}
V=c \sqrt{1-\frac{\omega_{c}^{2}}{\omega^{2}}}<c \\
E=\frac{m_{0} c^{2}}{\sqrt{1-\frac{V^{2}}{c^{2}}}}=\frac{\hbar \omega_{c}}{\frac{\omega_{c}}{\omega}}=\hbar \omega \\
p=\frac{m_{0} V}{\sqrt{1-\frac{V^{2}}{c^{2}}}}=\frac{\frac{\hbar \omega_{c}}{c^{2}} c \sqrt{1-\frac{\omega_{c}^{2}}{\omega^{2}}}}{\frac{\omega_{c}}{\omega}}=\hbar \beta
\end{gathered}
$$

Group velocity

$$
V_{p}=\frac{\omega}{\beta}=\frac{E}{p}=\frac{c}{\sqrt{1-\frac{\omega_{c}^{2}}{\omega^{2}}}}>c
$$

Energy velocity [8]

$$
V_{g}=\frac{d \omega}{d \beta}=c \sqrt{1-\frac{\omega_{c}^{2}}{\omega^{2}}}
$$

$$
\begin{gathered}
V_{e}=c \sqrt{1-\frac{\omega_{c}^{2}}{\omega^{2}}}<c \\
V_{p} \cdot V=V_{p} \cdot V_{e}=V_{p} \cdot V_{g}=c^{2}
\end{gathered}
$$

By comparison, the dispersion relation of high frequency EM waves in plasma is [26]

$$
\omega^{2}=k^{2} c^{2}+\omega_{p}^{2} \quad\left(\omega_{p}=\sqrt{\frac{N Z e^{2}}{\varepsilon_{o} m^{*}}} \text { plasma frequency }\right)
$$

Obviously, it is a good model to study de Broglie's theory because $E=\hbar \omega$ and $p=\hbar \mathrm{k}$ are both valid.

Total energy

Momentum

$$
E=\hbar \omega
$$

$$
p=\hbar k
$$


Rest mass

Mechanical velocity

$$
m_{0}=\frac{\hbar \omega_{p}}{c^{2}}
$$

$$
\begin{gathered}
V=c \sqrt{1-\frac{\omega_{p}^{2}}{\omega^{2}}}<c \\
E=\frac{m_{0} c^{2}}{\sqrt{1-\frac{V^{2}}{c^{2}}}}=\frac{\hbar \omega_{p}}{\frac{\omega_{p}}{\omega}}=\hbar \omega \\
p=\frac{m_{0} V}{\sqrt{1-\frac{V^{2}}{c^{2}}}}=\frac{\frac{\hbar \omega_{p}}{c^{2}} c \sqrt{1-\frac{\omega_{p}^{2}}{\omega^{2}}}}{\frac{\omega_{p}}{\omega}}=\hbar k
\end{gathered}
$$

Phase velocity

Group velocity

$$
V_{p}=\frac{\omega}{k}=\frac{E}{p}=\frac{c}{\sqrt{1-\frac{\omega_{p}^{2}}{\omega^{2}}}}>c
$$

$$
V_{g}=\frac{d \omega}{d k}=c \sqrt{1-\frac{\omega_{p}^{2}}{\omega^{2}}}
$$

Energy velocity [27]

$$
\begin{gathered}
V_{e}=c \sqrt{1-\frac{\omega_{p}^{2}}{\omega^{2}}}<c \\
V_{p} \cdot V=V_{p} \cdot V_{e}=V_{p} \cdot V_{g}=c^{2}
\end{gathered}
$$

The opinion is wrong that the frequency of de Broglie's matter wave has no physical effects [28][29] and only the frequency difference is important [30].

\section{CONCLUSION}

de Broglie's $\mathbf{p}=\hbar \mathbf{k}$ is a special case of $\mathbf{p}=\hbar \boldsymbol{\beta} . \quad\left[q,-i \hbar \frac{\partial}{\partial q}\right]$ equals $i \hbar$ indeed but the canonical commutation relation $[q, \hat{p}]=i \hbar$ is invalid because the operator $-i \hbar \frac{\partial}{\partial q}$ is not always a momentum.

\section{References}

[1] Trigg, G. L., Crucial Experiments in Modern Physics (New York: Van Nostrand Reinhold Co.,1971), Chap.9\&10

[2] Elliott, R. S., Antenna theory and design (revised ed.) (New Jersey: Wiley- Interscience,2003) pp. $440-453$

[3] Zhang, K and Li, D, Electromagnetic Theory for Microwaves and Optoelectronics (2nd ed.) (New York: Springer,2008) pp.401-472

[4] Jackson, J. D, Classical Electrodynamics (3rd ed.) (New York: John Wiely \& Sons, 1999) pp.361-364 
[5] Griffiths, D. J, Introduction to Electrodynamics (3rd ed.) (New Jersey: Prentice Hall,1999) pp.409-410

[6] Jackson, J. D, Classical Electrodynamics (3rd ed.) (New York: John Wiely \& Sons, 1999) pp.259-262

[7] Griffiths, D. J. Introduction to Electrodynamics (3rd ed.) (New Jersey: Prentice Hall,1999) pp.347-355

[8] Jackson, J. D. Classical Electrodynamics (3rd ed.) (New York: John Wiely \& Sons, 1999) pp.364

[9] Casimir, H. B. G. C, "On the attraction between two perfectly conducting plates", Proc. Kon. Nederland. Akad. Wetensch. B51, 793-795(1948)

[10] Spamaay, M. J, "The measurement of the Casimir force between two parallel metal plates", Physica. 24, 751-760 (1958)

[11] Lamoreaux, S. K, "Demonstration of the Casimir Force in the 0.6 to $6 \mu \mathrm{m}$ Range", Phys. Rev. Lett. 78, 5-8 (1997)

[12] Bressi, G, Carugno, G, Onofrio, R and Ruoso, G, "Measurement of the Casimir force between Parallel Metallic Surfaces", Phys. Rev. Lett. 88 , 041804 (2002)

[13] Wang, Z. Y, “Graphene, neutrino mass and oscillation”, arXiv:0909.1856v2 [physics.gen-ph]

[14] Bugaev S P, Cherepenin V A, Kanavets V I, Klimov A I, Kopenkin A D, Koshelev V I, Popov V A and Slepkov A I, "Relativistic multiwave Cherenkov generators", IEEE Trans . Plasma Science. 18, 525-536(1990)

[15] Zhang, K and Li, D, Electromagnetic Theory for Microwaves and Optoelectronics (2nd ed.) (Beijing: Publishing House of Electronics Industry Press,2001) pp.321(in Chinese)

[16] Goubau, G, "Surface waves and their application to transmission lines", J.Appl. Phys. 21, 1119-1128 (1950)

[17] Grace, A. C and Lane, J. A, "Surface-Wave Transmission Lines", Wireless. Engineer. 29, 230-231 (1952)

[18] Wang, K and Mittleman, D. M, "Metal wires for terahertz wave guiding”, Nature 432, 376379 (2004)

[19] Royer, D and Dieulesaint, E, Elastic Waves in Solids II: Generation Acousto-optic Interaction Applications (2nd ed.) (New York: Springer, 2000) pp.178

[20] Griffiths, D. J, Introduction to Quantum Mechanics (2nd ed.) (New Jersey: Prentice Hall, 2004), Chap. 2.6; 2.33

[21] Jackson, J. D, Classical Electrodynamics (3rd ed.) (New York: John Wiely \& Sons,1999) pp.363

[22] Greiner, W, Classical Electrodynamics (New York: Springer,1998) pp.366-369

[23] Feynman, R. P, Leighton, R. B and Sands, M, The Feynman lectures on physics (Vol.2) ( Reading: Addison-Wesley,1964) 24.29 24.31

[24] Jackson, J. D, Classical Electrodynamics (3rd ed.) (New York: John Wiely \& Sons,1999) pp.600-603

[25] Jackson, J. D Classical Electrodynamics (3rd ed.) (New York: John Wiely \& Sons,1999) pp.5-9

[26] Jackson, J. D, Classical Electrodynamics (3rd ed.) (New York: John Wiely \& Sons,1999) pp.313 
[27] Chen, H. C, Theory of Electromagnetic Waves: a coordinate-free approach (New York: McGraw-Hill,1983) 7.100 7.101

[28] Guan, H, Fundamental Concepts of Quantum Mechanics (Beijing:Higher Education Press, 1991) pp.70-71(in Chinese),

[29] Wichmann, E. H, Quantum Physics(Berkeley Physics Course Vol4) ( New York: McGrow Hill,1971) pp.272-274

[30] Pauli, W, Wave Mechanics(Pauli Lecture on Physics Vol5), (Cambridge: M.I.T. Press,1973) $\S 2$ 